Analysis of the biocompatibility and time of absorption of swine collagen membranes in surgical procedures

Reis, Q.N.; Magalhães, D.; Menezes, H.H.M.; Horbylon, B.Z.; Borges, D.C.; Gomes, C.F.; Carneiro, K.F.

The application of physical barriers for regeneration of bone defects was first proposed by Dahlin et al. (1988), who reported a technique known as guided bone regeneration (GBR). Currently, the principles of GBR have been used in the repair of bone defects; bone volume increase of resorbed alveolar ridges; development of bone areas for dental implants; associated or not with grafting procedures for post-extraction treatment of dental sockets for immediate implantation; treatment of periimplant fenestrations and dehiscence defects; and treatment of periimplantitis. In this way, GBR has been successfully used to treat some types of bone defects. Problems associated with the procedure, such as premature exposure of the membranes to ora cavity and the consequent contamination. The material of the swine membrane is a bone tissue matrix from the femur of pigs. Swines, including the mini-pigs, present similarities with humans with respect to dental aspects, relative to their morphology, physiology and biocompatibility. The purpose of this work was to provide information on the applicability, biocompatibility and time of absorption by the human organism of swine collagen membrane in regenerative periodontal procedures.

\section{Oral Rehabilition}

\section{Bond strength of denture teeth to acrylic resins after microwave irradiation}

Moffa, E.B.; Ribeiro, R.C.; Vergani, C.E.; Pavarina, A.C.; Izumida, F.E.; Giampaolo, E.T.

One of the most usual types of failure in a denture is bond failure between a denture tooth and an acrylic resin denture base. Thus, this study evaluated the bond strength of denture teeth (Vivodent-DV and Biotone-DB; central incisors) to acrylic resins QC20-QC, Acron MC-AC and Lucitone 550-L) after microwave irradiation. A total of 240 specimens were fabricated, 120 from each brand of denture tooth. All specimens were randomly divided into 4 groups $(n=10)$ : $\mathrm{G} 1$ (control group) immersed in distilled water at $37^{\circ} \mathrm{C}$ for $48 \mathrm{~h}, \mathrm{G} 2$ - immersed in distilled water at $37^{\circ} \mathrm{C}$ for $48 \mathrm{~h}$ and microwaved twice at $650 \mathrm{~W}$ for $6 \mathrm{~min}, \mathrm{G} 3$ - immersed in distilled water at $37^{\circ} \mathrm{C}$ for $48 \mathrm{~h}$ and microwaved daily for 7 days, G4 - immersed in distilled water at $37^{\circ} \mathrm{C}$ for 8 days. Shear load was applied with a load testing machine at 45 degrees from the long axis of each denture tooth on the palatal surface at a crosshead speed of $0.5 \mathrm{~mm} / \mathrm{min}$ until fracture. Student's test $(\mathrm{p}=0.05)$ was applied to data for the detection of significant differences between two brands of denture teeth. Most of bond strength values established between DB and the resins were significantly higher than those of DV, except for G1 and G2. For G1, the strength of QC bonded to DV was significantly higher $(\mathrm{P}<0.05)$ than $\mathrm{QC}$ bonded to $\mathrm{DB}$. No significant differences were observed with both brands of teeth when bonded to AC resin for G1 or bonded to L resin for G1 and G2. However, the variation (\%) of bond strength was predominantly lower with DB than DV. In conclusion, DB may be indicated with reliability due to its uniform behavior.

Misalignment of implant-supported prosthesis in relation to dental arch: 12 years of follow-up

Coro, V.; Júnior, C.D.S.; Neves, F.D.

The purpose of this study was to warn the dental community about a possible problem in partial implant-supported prosthesis for long periods in function. The misalignment between natural teeth and implant-supported prosthesis on teeth 11 and 12, observed in a 12-year clinical follow-up, illustrates the fact. Two Nobel Biocare ${ }^{\circledR}$ implants were placed and left covered for 6 months. The second-stage surgery was performed and the provisional denture was readapted. After 11 days, the abutments were installed and the fabrication of the prosthesis started. The definitive metal-ceramic crowns were placed in 2005, after a rigorous occlusal adjustment. Evaluations were made at 2, 4, 6, 9 and 12 years, when it could be noticed that the restorations were positioned palatally and with an extrusion in comparison to the natural teeth. The implants presented a natural bone loss to the system utilized. However, after 9 years, a greater discrepancy could be noticed, with anterior occlusion and esthetic changes. The possible causes have been discussed: occlusal problems, parafunctional habits and natural movement - strength anterior component. The two first options were discarded after clinical analysis and diagnosis. Therefore, the natural movement, probably deriving from an interaction of mechanical and genetic factors might have been the cause. However, further investigation is needed. The implants do not have periodontal ligaments, but rather an ankylosis, so they do not suffer those movements. This case emphasizes the need to inform the patients that the implants can last more than 10 years in function, but it is not the same with the restorations, when loosing function and esthetic, must be replaced, giving back harmony to the stomatognathic system.
Effect of thermocycling on the impact strength of one denture base and two reline acrylic resins

Bochio, B.C.; Lazarin, A.A.; Perez, L.E.C.; Vergani, C.E.; Pavarina, A.C.; Wady, A.F.; Machado, A.L.

Adequate impact strength is essential for relined dentures to resist the forces of mastication, thus preventing denture fracture. Clinically, the denture base materials are submitted to temperature changes during the intake of hot and cold food and drinks or the cleansing regimens. This study evaluated the effect of thermocycling on the impact strength of one heat-polymerizing resin (Lucitone 550 -L) when relined using the same material or the autopolymerizing reline resins Ufi-Gel Hard (U) and Tokuyama Rebase II (T). The impact strength of intact resin L specimens, before and after thermocycling, was also evaluated. Specimens of $\mathrm{L}(\mathrm{n}=30)$ were made $(60 \mathrm{~mm} \times$ $6 \mathrm{~mm} \times 2 \mathrm{~mm}$ ) and relined $(2 \mathrm{~mm})$ using the same material $(\mathrm{L} / \mathrm{L})$ or the relining resins $\mathrm{T}(\mathrm{L} / \mathrm{T})$ and $\mathrm{U}(\mathrm{L} / \mathrm{U})$. Intact $\mathrm{L}$ specimens $(\mathrm{n}=10)$ were also made $(60 \mathrm{~mm} \times 6 \mathrm{~mm} \times 4 \mathrm{~mm})$. The specimens were then divided into 2 groups: G1 (control - without thermocycling); G2 - specimens were thermally cycled for 5.000 cycles between $5 \pm 2^{\circ} \mathrm{C}$ and $55 \pm 2^{\circ} \mathrm{C}$. A $0.8 \mathrm{~mm} \mathrm{V-notch}$ was prepared and the specimens were submitted to the impact test (Charpy) using a $0.5 \mathrm{~J}$ Charpy pendulum and distance of $50 \mathrm{~mm}$ between the supports. Data $(\mathrm{kJ} / \mathrm{m} 2)$ were analyzed by Kruskal-Wallis test (á=0.01). For both groups, resin L (G1- 1.65; G2- 1.53) exhibited a significant increase in the mean impact strength after relining with $\mathrm{T}$ (G1-5.90; G2-4.71), a decrease after relining with U (G1-0.67; G2-0.68), and remained unaffected after relining with L (G1-1.71; G2-1.54). Only L/L combination (1.71) showed a decrease after thermocycling (1.54). Thermocycling had no deleterious effect on the impact strength of the specimens relined with materials $\mathrm{T}$ and $\mathrm{U}$, but promoted a decrease when relining was made with resin L. The highest impact strength was observed for $\mathrm{L} / \mathrm{T}$ combination.

Impact strength of a heat-polymerizing denture base acrylic resin and two autopolymerizing reline resins

Wady, A.F.; Lazarin, A.A.; Perez, L.E.C.; Vergani, C.E.; Giampaolo, E.T.; Bochio, B.C.; Machado, A.L.

As denture wearers become older and lose much of their supporting alveolar bone, the need for continuing prosthodontic care such as relines is more evident than previously. The relining procedure may affect the impact strength of the denture base and lead to the fracture of the removable prosthesis. This study evaluated the impact strength of one heat-polymerizing denture base resin (Lucitone 550-L) and two autopolymerizing reline resins (Tokuyama Rebase-T and Ufi Gel Hard -U). The impact strength of specimens of resin $L$ relined with the same material and with the autopolymerizing relining resins was also evaluated. Specimens of $L(n=18)$ were made $(60 \mathrm{~mm} \times 6 \mathrm{~mm} \times 2 \mathrm{~mm})$ and relined $(2 \mathrm{~mm})$ with the same material $(\mathrm{L} / \mathrm{L})$ or with the relining resins $\mathrm{T}(\mathrm{L} / \mathrm{T})$ and $\mathrm{U}(\mathrm{L} / \mathrm{U})$. In addition, intact $\mathrm{L}, \mathrm{T}$ and $\mathrm{U}$ specimens $(\mathrm{n}=6)$ were made $(60 \mathrm{~mm} \times 6 \mathrm{~mm} \times 4 \mathrm{~mm})$. All specimens were prepared according to the manufacturers' instructions, and a V-notch was then cut to a depth of $0.8 \mathrm{~mm}$. The impact test (Charpy) was carried out with a pendulum rated at $0.5 \mathrm{~J}$ and distance of $50 \mathrm{~mm}$ between supports. Data $\left(\mathrm{kJ} / \mathrm{m}^{2}\right)$ were analyzed by Kruskal-Wallis test (á=0.01), which indicated that the mean impact strength of resin $\mathrm{L}$ (1.72) was higher than that of resin $\mathrm{U}(0.86)$, which in turn was higher than that of material $\mathrm{T}(0.69)$. There was no significant difference between L (1.72) and L/L combination (1.72). Compared to the intact resin $\mathrm{L}$, the mean impact strength was significantly increased after relining with $\mathrm{T}$ (L/T-6.45), whereas relining with $\mathrm{U}$ significantly decreased the mean impact strength ( $\mathrm{L} / \mathrm{U}-0.67)$. The impact strength of resin $\mathrm{L}$ remained unaffected after relining with the same material, and was significantly increased after relining with resin $\mathrm{T}$.

\section{Evaluation of phonetics in prosthodontic patients}

Hilgenberg, P.B.; Saldanha, A.D.D.; Silva, P.M.B.; Calderon, P.S.; Porto, V.C.

Dental procedures must respect esthetics, phonetics, masticatory and deglutition functions, oral functions and the neuromuscular system. The purpose of the present study was to make a literature review of the most important aspects of speech related to dental prostheses. Furthermore, a speech conference list was proposed to be used during dental treatment. Speech is the most complicated response of men, but it is frequently underestimated because its complexity is not apparent. Speech rehabilitation can be done by the reshaping of teeth and palate of the dental prosthesis, reestablishment of the vertical dimension, free functional space and closest speaking space. The sounds are described by the way they are produced and by the articulators they involve. They can be classified as: plosive, fricative, nasal, liquid, affricative, basal and vibrating. For dental procedures, during denture adjustment, the most important sound is $/ \mathrm{s} /$. The correct production of speech might be altered when there is anterior open bite, mispositioned teeth position, tooth loss, inadequate tooth shape and anatomy, alteration in vertical dimension, in free functional space and closest speaking space, discrepancy between overjet and overbite. The two most common speech errors are lisping and whistling. They are characterized as escape of air with substitution of certain sounds and whistle, respectively. All dentists must 
be aware of these events not to install a new dental prosthesis with new speech sensations or alterations. To the correct production of sound, it is mandatory that all speech articulators and the stomatognathic system are well-balanced.

\section{Differential diagnosis in the treatment of orofacial pain}

Silva, P.M.B.; Calderon, P.S.; Saldanha, A.D.D.; Hilgenberg, P.B.; Rodrigues, K.R.; Conti, P.C.R

The dentist is the professional involved in the diagnosis and management of orofacia pains. The diagnosis, however, can be quite difficult, due to the fact that many painful disorders of non-odontogenic origin can refer pain to the teeth, which frequently lead to wrong treatment strategies. In this scenario, myofascial and neuropathic pain conditions are extremely important. The purpose of this work was to demonstrate, by a case report, the importance of the differential diagnosis in order to determine the origin of pain in orofacial pain patients. The case refers to a 43 year-old female patient, presenting a clinical situation of pain on the left side of the face (supra orbital and lateral to the nose), including the maxillary teeth area at the same side. In this case, the correct diagnosis and treatment were established after the accomplishment of different exams and specific diagnosis tests. The dental professional should be aware of the differential diagnosis because irreversible and expansive treatments are inadequately used in the control of pain of different origins, with economic and biologic consequences to the patient.

\section{Pharmacotherapy in the management of chronic orofacial pain}

Saldanha, A.D.D.; Hilgenberg, P.B.; Silva, P.M.B.; Calderon, P.S.; Vedolin, G.M.; Conti, P.C.R.

Orofacial pains may arise from musculoskeletal, neuropathic, (neuro) vascular, visceral and/or psychiatric origins. Drug selection depends on a variety of factors with the most important factor being an accurate diagnosis. Pharmacotherapy is a cornerstone in the treatment of pain and should be directed towards the source of pain. Various drugs are recommended in the management of orofacial pain as nonopioid and opioid analgesics, corticosteroids, skeletal muscle relaxants, anticonvulsants, antidepressants and anxiolytics. Generally, symptoms of depression, sleep disturbance, stress and drug dependency may accompany chronic pain and must be treated concomitantly, associated psychological therapy.

\section{“Adhesive Endo Crown” as a therapeutic resource for endodontically treated teeth}

Costa, Y.M.; Mondelli, J.; Sábio, S.S.; Ido, V.Y.; Sábio, S.

This study suggests an innovative form of therapeutics as restoration for endodontically treated teeth with great destruction of the coronary remainder. The conventional techniques promote the reconstruction of these teeth with cast metal cores, prefabricated cores or filling cores associated with partial or full crowns depending on the extension of coronal destruction. These treatments have provided quite satisfactory clinical outcomes over time, which have determined their acceptance among the dental class. However, these treatments are expensive and demand several operative stages. In 1999, Bindl, Mörmann presented a therapeutic approach denominated Endo Crown, which proposed the treatment of these teeth in a single session by fabrication of adhesive crowns using the Cerec system. This report suggests modifications adapting to the indirect restorative system materials of laboratorial use that are more accessible and available to the clinicians, such as modified porcelains or laboratory resins. The modified restorative system presents good clinical perspectives from the functional and esthetic points of view, because it adapts to the conditions of most laboratories and, for this reason, the costs are more accessible to the dentist and patient. However, given the small number of reported cases, it is advisable that indication of this treatment is made only based on well selected clinical situations.

Rehabilitation by mesial cantilever for preservation of the integrity of the canine: a case report

Júnior, F.A.A.; Bezzon, O.L.

A favorable treatment to substitute the loss of tooth is the use of a fixed partial denture (FPD) that has both sides supported. The use of distal cantilever is considered an exception because, especially in unilaterally edentulous dentition, it is preferable in relation to removable partial denture (RPD). The mechanics of cantilever FPD requires the abutment adjacent to the replacement tooth to possess suitable periodontal support (Antonoff SJ, 1973) because the greatest functional stress is directed on the nearest abutment (Henderson D et al., 1970). Ewing (1957) cited the following requirements to use of cantilevered PDF: sound periodontal support, satisfactory root morphology and a favorable arch-to-arch relationship. This study report the case of a patient with absence of tooth 24, having teeth 25 and 26 compromised by extensive, unsatisfactory restorations and unfavorable oclusal plain, however, with tooth 13 completely sound. After clinical-radiographic examination and analysis of the study models mounted in a semi-adjustable articulator, the treatment plan was settled including the fabrication of mesial cantilevered fixed metal ceramic partial denture for replacement of tooth 24 , having teeth $25 / 26$ as abutments; such planning objectified preservation of 13 . In this case the use of implants was not accepted by the patient. In these situations, the usual treatment choices are cantilever FPDs or RPRs. Although both types of treatment can recover the patient function, the fixed denture, in this case, was better accepted, also for allowing the regularization of the Spee curve. From retrospective studies, it may be concluded that, in spite of new possibilities for implants for the extension of shortened arches or for replacement of anterior teeth, the use of cantilevered restoration to replace a dental loss presents good prognosis and can still be indicated as a safe and simple technique with good longevity.

Oclusal splint and low-level laser in the control of pain orofacial and in the evaluation of the functional mobility of the lumbar spine

Ferreira, L.M.A.; Fagundes, K.S.; Videira, A.C.T.; Hotta, T.H.; Regalo, S.C.H.; Bataglion, C.

Physical therapy has currently an important role in functional recovery of patients with temporomandibular disorders (TMD) during clinical and physical evaluation. To help during treatment, low-level laser therapy (LLLT) has been used as an auxiliary resource in pain control. The aim of this study was to demonstrate the importance of the LLLT with occlusal splint appliance, in improving pain control and functional mobility in lumbar spine of 7 patients treated at the "Clínica de Disfunção Temporomandibular e Dor Orofacial em Pacientes Com Necessidades Especiais: Abordagem Mulidisciplinar", of the School of Dentistry of Ribeirão Preto, University of São Paulo (Research Ethics Committee no. 2006.1.411.58.0). An Analogical Visual Scale (AVS) was used together with and postural physical therapy evaluation as initial and final evaluation instruments. The proposed treatment was the association of occlusal splint and 10 sections of LLLT (AlAsGa; 780nm wavelength, $70 \mathrm{~mW}$ power, $35 \mathrm{~J} / \mathrm{cm}^{2}$ dosimetry). Based on the obtained results, it may be concluded that the therapies used in this study were effective in eliminating orofacial pain and improving lumbar mobility.

\section{Prosthetic rehabilitation in a patient with pycnodysostosis}

Rezende, M.S.; Oliveira, A.C.S.; Carvalho, P.M.; Guimarães, R.A.P.; Brazão-Silva, M.T.; Marra, D.T.; Costa, M.M.

The pycnodysostosis (PYCD) is a rare skeletal recessive autosomal dysplasia in consequence of altered codification gene of catepsina K enzyme. Moroteaux and Lamy first described this alteration in 1963, which may also be called MaroteauxLamy syndrome or mucopolysaccharidosis type VI. The main clinical manifestations include low stature of patients, micrognathia, and delay, malformation or mispositioning of teeth. Additionally, osseous fragility with history of pathologic fractures can often be found. Osteomyelitis represents the most aggravator of complications in consequence of oral problems, being more common in adults that in children. Although clinical and radiographic aspects of this syndrome are well established in literature, reports concerning the oral rehabilitation of these patients are rare. Based on this, this work reports a case of a female patient diagnosed with PYCD emphasizing the oral findings, and describing a supportive treatment comprising patient oral rehabilitation. Occlusal rearrangement was undertaken by fabrication of an overdenture. The outcomes were extremely satisfactory and the treatment was conservative and allowed the establishment of a stable occlusion. Tooth position was not altered, but the inter-arch relationship was improved, provide occlusal stability. This type of prosthesis permits easy cleansing and use in PYCD patients with proper case selection, representing an efficient and safe alternative for rehabilitation of these patients.

The use of magnetic retention as an option for prosthetic rehabilitation of patients with periodontal disease

Leite, P.H.A.S.; Guerra, C.M.F.; Moraes, S.L.G.; Bezerra, C.F.R; Neto, A.F.; Carreiro, A.F.P.

The magnetos have been constantly used in Dentistry due to their multiple applications, mainly in the prosthetic area and some orthodontic situations. The use of magnetos in removable partial denture becomes a valid alternative to promote retention and stability, eliminating prosthetic structures that can affect esthetics and mainly reducing tensions on periodontium. That way, they are an excellent indication for patients who present supporting teeth with reduced periodontal support due to bone loss secondary to periodontal disease, promoting a better retention and reducing 
the transmission of extreme forces to these structures. The magnetos have a simple technique and are easily incorporated to the denture, being the most used the Nd-Fe$\mathrm{B}$ as well as those which associate cobalt and platinum. One of its limitations is its low resistance to corrosion by oral fluids, needing to be coated by another metal, usually titanium. The purpose of this study was to present an alternative treatmen for the prosthetic rehabilitation of the pillar teeth with periodontal disease, highlighting the functional, biological and esthetic advantages of the use of magnetos in removable partial denture (overdentures).

Benefits of artificial gingiva in a patient with unfavorable labial line and rehabilitated with implant-supported fixed partial denture

Marra, D.T.; Lima, J.H.F.; Carvalho, P.M.; Oliveira, A.C.S.; Brazão-Silva, M.T.; Diniz, P.V.P.; Costa, M.M

The rehabilitation of patients with implant-supported fixed dentures improves the masticatory performance, and provides more satisfactory results when compared to others dentures. However, the esthetics in some situations is not fully achieved, mainly in cases of accentuated bone losses and unfavorable labial line. In these cases, it is necessary to seek alternatives to meet patients' requirements and expectations. A viable alternative has been the use of artificial gingiva to promote acceptable esthetics without compromising the cleaning cervical areas of implantsupported dentures. The aim of this work was to report the case of a patient with large exposure of the cervical region of the implants due to little bone availability and unfavorable smile line. In attempt to overcome these problems, a removable artificial gingiva was fabricated and the final results were extremely satisfactory. In conclusion, with proper case selection, this is an additional artificial method to improve the success of this type of prosthesis.

\section{Effect of microwave disinfection on toothbrush wear resistance of denture base} resins

Cintra, A.V.; Izumida, F.E.; Ribeiro, R.C.; Machado, A.L.; Vergani, C.E.; Pavarina, A.C.; Giampaolo, E.T.

This study investigated the wear of Lucitone 550 and QC 20 denture base resins after microwave disinfection. Twenty specimens of each material were prepared (40x20x2mm). The specimens were submitted to successive weighing until constant mass. The specimens were placed in a toothbrush machine with deionizer water/ dentifrice slurry at 1:1 ratio. Each specimen was brushed in a toothbrush abrasion machine using 20,000 brushstrokes. A weight of 200g was applied to the brushes during the test. The specimens were weight again and the abrasion was calculated $(\mu g)$ by the difference of initial and final weight. Prior to toothbrushing, the specimens were divided into 2 groups: G1 (control group)- immersed in distilled water for 48 hours and G2 - immersed in distilled water for 48 hours and submitted to 2 sixminutes microwave disinfection cycles at $650 \mathrm{~W}$. This procedure simulated denture disinfection before laboratorial adjustments to prevent cross contamination. The results were subjected to ANOVA, Levene and Shapiro Wilk test (á=0.05). There was no significant difference between the experimental and the control groups. In conclusion, loss of mass of denture resins was not influenced by microwave disinfection.

Acting prosthesis for reestablishment and diagnosis of diminished vertica dimension of occlusion

Diniz, P.V.P.; Oliveira, A.C.S.; Arantes, F.N.; Brazão-Silva, M.T.; Rezende, M.S.; Marra, D.T.; Prado, C.J.

The rehabilitation of individuals with diminished vertical dimension of occlusion (VDO) is still a great challenge to dentists. Firstly, it is necessary to make a correct diagnosis. Clinical situations in which there are few and severely destroyed remaining teeth and irregular occlusal plane with reduced VDO present esthetic, chewing, phonetic deficiencies, usually associated with fibromyalgia. Therefore, the foremos steps are to reestablish these functions, especially VDO, and to evaluate them during a certain period. Then, the definitive rehabilitating treatment can be planned only after the dentist is sure that the "new" VDO is comfortable to the patient. An alternative to reestablish VDO, centric relation and recover stability and occlusal harmony is the use of overlay. This case report presents the clinical and laboratorial phases of the fabrication of this denture modality and discusses its advantages and indications. This type of denture is of easy and fast fabrication and has a high cost-effectiveness ratio.
Immediate reconstruction of a severely abraded dentition using an overlay: provisional and definitive treatment phases

\section{Júnior, A.C.F.; Oliveira, A.P.L.; Verde, M.A.R.L.; Rocha, E.P.; Silva, A.M.}

The oral rehabilitation in patients with severely abraded dentition has been a great challenge for the dentists, mostly for those less experienced. These teeth excessive worn are usually associated to parafunctional habits like bruxism, that is more prejudicial in association with others problems, like tooth erosion. The loss of vertical dimension of occlusion, the occlusal instability and the loss of anterior guidance occasioned by the excessive tooth wear might damage the stomatognathic apparatus' biology, function and esthetics. Then, a correct diagnosis and treatment planning of the case are crucial to control and reestablish the apparatus' harmony. This situation prompted studies to develop effective therapies for the treatment and control of parafunctional habits, which can involve the muscles, the temporomandibular joint and the teeth. Thus, the purpose of this report was to describe the therapy used in a patient with severely abraded dentition caused by bruxism associated with tooth erosion, with satisfactory bone support and gingival health, but with loss of vertical dimension of occlusion and posterior bite collapse. In the provisional treatment phase, a vertical dimension reestablishment plate was made to the immediate recovery of the function and esthetics. It is a reversible and no invasive treatment with a lower cost. After a 2-month surveillance period and the patient being asymptomatic, the definitive rehabilitation phase started, involving conjugated fixed and removable partial dentures. The patient's self-esteem recovered and he returned to his society circle.

\section{Biological and biomechanical aspects of the cone-morse implants}

Maior, B.S.S.; Filho, R.V.; Netto, B.P.; Assis, N.M.S.P.

In view of some discussed and demonstrated complications in the literature, especially problems of marginal bone resorption, loosening and fracture of screws, and aiming at increasing the stability in the implant-crown interface, new designs of prosthetic connections between crown and implant were introduced to the market to meet esthetic and functional demands. Implants with internal connection, with Morse taper characteristics provide a reinforced connection between implant and abutment, generating a cold welding among those elements, absence of microgap and increased resistance to micro-movements providing a rigid connection. The objective of this study was to demonstrate the biological and biomechanical behavior the implants and prosthetic components of the cone-morse system.

Acrylic resin removable gingiva: a simple and esthetic alternative to replace anterior periodontal tissues

Neto, A.F.; Amaral, B.A.; Branco, N.; Moraes, S.; Guerra, C.M.F.; Aquino, L.M.M.; Carreiro, A.F.P.

The use of conjugated fixed/removable partial dentures is an excellent alternative to solve esthetic and functional problems in Kennedy Class I patients. A 59-year-old patient came to the dental clinic of the Dental School of Ingá complaining of esthetic discomfort and functional difficulties. The intraoral examination revealed that teeth $16,17,18,26,27,28,34,35,36,37,38,46,47$ and 48 were missing. Case planning determined the fabrication of fixed dentures joining all the remaining teeth of the maxillary arch, which presented with poor esthetics due to staining and fractures, conjugated with a removable plug-in denture in the molar region. A conventional removable denture was constructed for rehabilitation of the mandibular arch. It may be concluded that this technique allows the dentist joining the high-quality esthetics of porcelain in the anterior region with the best force distribution provided by removable partial prostheses compared to cantilevered fixed dentures.

Analysis of relative artificial tooth position changes during specific upper denture processing phases by means of computer graphics

Sturion, L.; Ortolan, A.P.S.; Shibayama, R.

Artificial tooth position changes were investigated during upper complete denture processing. QC 20 (Dentsply) and Onda Cryl (Clássico - specific for microwave energy) resins were used and flasked in type II gypsum stone mould or silicone mould, polymerized in hot water bath as well in microwave polymerization. Forty specimens were used, which were obtained from one standard cast, and divided into four groups $(n=10)$ that were processed as follows: a) group I: flasked in metallic flasks with gypsum stone barrier and conventional polymerization. b) group II: flasked in glass fiber flasks with gypsum stone barrier and polymerization by microwave energy. c) group III: flasked in metallic flasks with silicone barrier and conventional polymerization. d) group IV: flasked in glass fiber flasks with silicone barrier and microwave polymerization. An acrylic resin guide was used to detect tooth displacements, this guide was marked with 5 predetermined points in teeth 
cusps that has proportionate reference points in the replicas. These points were used to measure, using AutoCad software, tooth displacements by digitized occlusa surface of the teeth. Based on the results and according to the employed methodology, the following can be concluded: 1 . Tooth position changes occurred after denture processing in all experimental groups. 2. The technique of flasking with silicone mould and microwave polymerization (group 4) presented minor degree of tooth position changes during all the processing. 3 . The segments that constitute the smaller triangle represented by the points of teeth 15, 21 and 25 showed more homogeneity and linearity than the larger triangle represented by the cusps of teeth 17, 21 and 27. 4. The use of silicone mould instead of gypsum stone mould for total denture flasking resulted in minor degree of dimensional changes regardless to the method of polymerization.

Effect of internal bleaching with $37 \%$ carbamide peroxide on fracture strength and failure pattern of teeth reconstructed with different restorative procedures

Nápoles, B.B.; Kaizer, O.B.; Reis, K.R.; Calderon, P.S.; Silva, P.M.B.; Bonfante, G.

This study investigated the compressive fracture strength and failure pattern in premolars submitted to endodontic treatment and internal bleaching with 37\% carbamide peroxide for 21 days, with application of different restorative procedures. Six groups were employed $(n=10): 1$ ) non-bleached teeth and pulp chamber sealed with IRM; 2) bleached teeth and pulp chamber sealed with IRM; 3 ) bleached teeth and pulp chamber filled with light cured composite resin; 4) bleached teeth, root canals prepared at $10 \mathrm{~mm}$, filling of the root canals and pulp chamber with IRM; 5 ) bleached teeth, root canals prepared at $10 \mathrm{~mm}$, luting of prefabricated metallic pos with zinc phosphate and pulp chamber sealed with composite resin; 6) bleached teeth, root canals prepared at $10 \mathrm{~mm}$, luting of glass fiber post with resin cement and pulp chamber sealed with composite resin. After 24-hour storage in distilled water, the specimens were submitted to compressive fracture strength testing in a universal testing machine. The following values were found: Group 1 - 56.23kgf; Group 2 48.96kgf; Group 3 - 53.99kgf; Group 4 - 45.72kgf; Group 5 - 54.22kgf; Group 6 $-60.12 \mathrm{kgf}$. The analysis of variance did not reveal statistically significant difference between groups ( $p>0.05$ ), suggesting that internal bleaching with $37 \%$ carbamide peroxide did not weaken the dental tissues. The largest number of unfavorable fractures was observed for Groups 2 (50\%), 4 (40\%), and 5 (30\%). Group 6 exhibited the most favorable failure pattern. The results suggest that internal bleaching with $37 \%$ carbamide peroxide did not significantly weaken the teeth. Among the bleached teeth, those with temporary IRM restorations or metallic posts demonstrated the most unfavorable fracture pattern, whereas the most favorable fracture pattern occurred in teeth restored with composite resin and glass fiber posts.

Immediate complete denture: a clinical option in immediate rehabilitation of patients with advanced periodontal disease

Guimarães, R.A.P.; Gomes, V.L.; Miranda, P.; Oliveira, A.C.S.; Brazão-Silva, M.T.; Rezende, M.S.; Gonçalves, L.C.

Periodontal disease is a bacterial infection that affects the periodontal tissues of protection and support. The remaining teeth often show peccary and incompatible conditions with the facial esthetic, masticatory function and general health status of the patients. In addition, the social relations of these patients are commonly affected by the consequences of this disease. Immediate complete denture (ICD) represents a very important appliance for use between the time of full-mouth extraction and fabrication of definitive prosthesis. For diagnosis, the clinical interview associated with clinical and radiographic date and analyses of study models are indispensable. It is mandatory for treatment success that the patient be aware of all phases of treatment and be in accordance with them. A 33-year-old woman presented to dental care with significant amount of bacterial plaque, dental calculus, gingival inflammation and bleed with demanding gingival purulent collections. The teeth had high mobility, pain, caries and roots remnants. It was also observed buccal displacement of the teeth causing mouth breathing, and drying of lips and mucosa. Radiographically, a poor prognosis was evident to accentuated bone resorption. Because of the patient's low socioeconomic level together with her lack of motivation due to her oral conditions a treatment plan comprising full-mouth extraction and installation of an immediate complete denture wa settled. All phases of this case were documented. The goals of this work were to discuss the psychological influence of this treatment, treatment planning, fabrication and installation of the ICD and address its characteristics, advantages and disadvantages.

Electromyographic activity of the masseter and temporal muscles of individuals with complete denture, partially edentate and dentate

Hotta, P.T.H.; Hotta, T.H.; Vitti, M.; Vasconcelos, P.B.; Rosa, L.B.; Regalo, S.C.H.

Alterations in the masticatory performance and the activity of the masticatory muscles can occur due to tooth absence, inadequate conditions of remaining teeth or poorly adapted complete denture. The purpose of this study was to analyze the electromyographic activity of the right and left masseter and anterior temporal muscles (LT, RT, LM, RM) in different groups. Three groups were formed: group $1(\mathrm{n}=9)$ individuals with complete denture; group $2(n=9)$ - individuals with absence of at least 10 teeth; and group $3(n=9)$ - dentate individuals. The analysis was performed using a Myosystem Br-1 electromyographer to record the rest position, mandibular movement and bite force in molars. The means of the collected data were normalized by the maximum voluntary contraction ( 4 seconds) and the results were analyzed statistically using SPSS software. Analyzing the rest, the right laterality, left laterality, protrusion and bite force positions, the differences were significant at $\mathrm{p}<0.01$, group 2 presenting the highest electromyographic means. It may be concluded that group 2 had greater muscular staple fiber conscription for the execution of the requested activities, probably, for the inadequate oclusal conditions presented by this group.

Fabrication of provisional prosthesis with acetate matrix: a clinical alternative

Linares, L.; Oliveira, P.C.G.

For any type of prosthetic treatment, from a single-tooth denture up to an oral rehabilitation, the fabrication of provisional crowns is a basic condition for treatment success. This is due to their key role in the biological protection (pulp and periodontal) to tooth preparation, in addition to reestablishing favorable oral conditions (DVO, guides, chewing, esthetics), thus providing the necessary information for settlement of an effective definitive treatment. In this way, it is imperative to elaborate provisional prostheses with the same diligence as that of the definitive prostheses, recovering esthetics and function, without increasing the cost of the treatment to the patient. It is known that provisional prostheses fabricated by indirect (laboratorial) technique presents advantages over the direct techniques with respect to their greater esthetic durability and better finishing and burnishing, which minimizes plaque accumulation. However, the high cost is inevitable and, many times, can render the treatment impracticable. This work presents an alternative to the fabrication of provisional crowns, using a plasticized polypropylene matrix, known as mixed or hybrid technique for fabrication of provisional crowns. This technique has the advantages of the indirect technique, however, with lesser cost and maintenance of the efficiency of the rehabilitating treatment. It is fabricated from the duplication of the diagnostic waxing model, which corrects tooth position and shape. The polypropylene matrix is formed in a vacuum plasticizer and trimmed. Next, the autopolymerizing acrylic resin is prepared and packed into the matrix and placed into the patient's mouth, guaranteeing a provisional crown with better adaptation and with all the advantages mentioned above.

Differences in psychological factors, pain intensity level and associated characteristics in myofascial pain patients with and without migraine

Gonçalves, P.Z.; Silva, R.S.; Merrill, R.; Conti, P.C.R

Myofascial PAIN (MFP) has been associated with psychological impairment, especially depression and anxiety. Decreased function and increased disability have also been found. Headache, including migraine, is a common finding among these patients, and can be impacted by painful TMJ and facial muscles structures, as the pain pathways of both conditions are similar (trigeminal nerve). This work compared the differences in psychological factors, pain level and associated characteristics in myofascial pain patient with and without migraine. A total of 229 orofacial pain patients from UCLA Graduate Orofacial Pain Clinic with primary diagnosis of myofascial pain, according to the American Academy of Orofacial Pain (AAOP) criteria, were asked to complete the Beck Depression Inventory (BDI) and Beck Anxiety Inventory (BAI), as well as visual analogue scale (VAS) questionnaires regarding pain intensity level (now, high, usual, low), mood level, disability level due to the pain, interference with jaw function level, and quality of rest. Patients with migraine were diagnosed according to the International Classification of Headache Disorders, second edition (ICHD-II) of the International Headache Society (IHS). T-test was used to detect differences between groups at a level of significance of 5\%. From a total of 229 MFP patients, 197 were female (86\%), and 71 (31\%) had an additional diagnosis of migraine (MFP + migraine group). The significance level adopted was $5 \%$. The MFP + migraine group presented higher levels for all variables in comparison to the MFP only group. However, only for anxiety ( $\mathrm{p}=0.04 \%$ ), highest pain intensity $(\mathrm{p}=0.01 \%)$ and quality of rest $(\mathrm{p}=0.01 \%)$ those differences were statistically significant. MFP patients with migraine had higher level anxiety, highest pain intensity and poorest rest comparing to MFP only patients. Further research is needed to clarify these differences. The authors wish to thank CAPES for the financial support. 
Immediate rebase of class I removable partial denture: case report

Nery, K.F.; Oliveira, J.L.G.; Bonfante, G.; Valle, A.L.; Rubo, J.H.; Somensi, D.S.; Oliveira, P.C.G.

The rehabilitation of Kennedy Class I patients, employing remove partial dentures (RPD), is a very great challenge for the dentists, mainly concerning the posterior control of the prosthesis, because over the years, there is a tendency to loosen the adaptation from the basis of the prosthesis in relation to the alveolar ridge, due to the physiologic process of bone resorption. There are several factors related to the bone resorption process from systemic problems of the patients until imperfections on the prosthesis basis to the ridge may result in instability of the prosthesis, alterations on the centric occlusion, vertical dimension of occlusion reduction, metallic structure, loss of adaptation, occlusion strength, excess on the anterior teeth, TM alterations, tissues traumas and impaired mastication this way, recognizing that bone resorption is a know process and also irreversible, it becomes indispensable the performance of some procedures for the posterior control of these prostheses and one of these includes the performance of periodic rebase. Rebase is a clinical and laboratorial procedure employed to recover the prosthesis basal surface to the residual ridge, by filling of these structures with appropriate material. Thus it is possible to maintain the occlusal fitness, to favor the retention and stability to avoid damage to the tissues, to recover masticatory efficacy, avoid food accumulation between the prosthesis basis and the ridge, to eliminate the discomfort of lack of stability and favor the cleansing. This way, the objective of the present work was to describe the rebase procedure from the basis of a RPD presenting edentulous ridge by the immediate technique which is applied directly on the oral cavity by use of autopolymerizing acrylic resin.

Assessment of bond strength of thermally activated acrylic resin teeth after the action of ultrasound and disinfecting solutions

Ribeiro, A.B.; Mian, H.; Sato, S.; Silva, F.B.; Pedrazzi, V.

All prosthetic pieces must be carefully treated in order to maintain their biosafety and prevent cross-infections. On the other hand, the influence of physical and chemical treatment on bond strength of artificial teeth to acrylic denture bases is still a challenge. The purpose of this study was to compare the shear bond strength of double- and triple-layer artificial teeth (Biolux ${ }^{\otimes}$ and Trilux ${ }^{\circledR}$ ) after the action of disinfecting solutions (Listerine ${ }^{\circledast}$, Cepacol ${ }^{\circledR}$, Periogard ${ }^{\circledast}$, enzymatic crust remover Riozyme II $^{\circledR}$ and $1 \%$ sodium hypochlorite and tap water, as a control) plus ultrasonication. Seventy-two specimens from each tooth type were obtained and included in cylindrical heat polymerized resin bases, originating 12 groups. Six of those groups were ultrasonicated with disinfectants for 4 cycles, 15 minutes each; the other 6 groups were immersed in the solutions for the same period of time. Bond strength means were obtained by means of a shearing test in a 2000 DL universal testing machine and the results were converted into MPa. The double pressed acrylic teeth presented shear bond strength means of $3.43 \mathrm{MPa}$, while the triple pressed acrylic teeth had shear bond strength means of 3.02 MPa. Statistical analysis using ANOVA and Fisher's LSD multiple-comparison test confirmed that triple pressed acrylic teeth presented lower bond strength when submitted to disinfectants in both assayed situations in comparison to the double pressed teeth. It may be conducted that ultrasonication and the tested disinfectants did not affect the bond strength of both types of studied acrylic teeth. The double pressed acrylic teeth presented higher shear bond strength than the triple pressed teeth.

\section{Application of a rotational removable partial denture on the dental clinic: case} report

Somensi, D.S.; Oliveira, J.L.G.; Rubo, J.H.; Valle, A.L.; Bonfante, G.; Nery, K.F.; Oliveira, P.C.G

The rotational removable partial denture is characterized by the presence of two different axis of insertion in one single piece. Thus, the rests are positioned in two steps that follow the two trajectories of insertion. The possibility of utilization of a lay in a rotational way to the removable partial denture has shown to be a great ally during the planning phase, mainly in the resolution of Kennedy's Class IV, due to the unfavorable esthetic conditions imposed by the retention clasps which, if removed in a randomly way, can cause both biomechanical and functional impairments to the set. Therefore, the main advantage of using the rotational insertion axis resides in the fact that some clasps can be eliminated without any influence in the basic mechanical requisites of the right retainer: retention, support and stability. Moreover, the lack of these clasps results in a better esthetic and in the reduction in the amount of components over the tooth. When compared to the fixed prosthesis, the rotational removable partial denture is preferable concerning the preservation of the tooth structure, regarding the less amount of tooth preparation. This type of removable partial denture also shows a huge advantage over the removable prosthesis with interlocks of precision and semi-precision due to an easier construction, more conservative approach and lower cost. The removal of the prosthesis is made by an inverse movement than the one employed during the insertion, which makes impossible the displacement in the vertical direction. This fact indicates the suitability of the rotational removable partial denture to palatal denture. Therefore, the main objective of this work was to demonstrate, by the report of a case, the particularities present in the planning of a rotational RPD and moreover, the esthetic result that this kind of prosthesis can provide.

Oral rehabilitation with implant-supported prostheses: a case report

Borges, A.F.; Rego, M.R.M.; Costa, L.C.S.; Mota, A.S.

A 67-year-old female patient presented with totally edentulous mandible and partially edentulous maxilla, with remaining teeth $13,12,11,21,22$ and 23 restored with metal ceramic restorations. These restorations were inadequate functionally and esthetically, with pronounced buccal inclination. Radiographically, images suggestive of root fracture were observed on teeth 11,12 and 13 , in addition to poor periodontal implantation of all six remaining teeth. During planning and diagnosis, it was decided for the extraction of the remaining teeth and for the installation of osseointegrated implants and immediate fixed prosthesis for the maxillary and mandibular arches. For determining the correct buccolingual position of the anterior prosthetic elements of the provisional restoration, a Steiner cephalometric tracing was used for positioning the central incisor because the remaining teeth were not a good esthetic or functional reference as they were buccally tipped. Thus, surgical procedures were accomplished for extraction of the maxillary anterior teeth and for immediate installation of the osseointegrated implants (Neodent ${ }^{\circledR}$ ) in the region of teeth 13, 11, 21 and 23. These implants presented initial stability higher then 35 $\mathrm{Ncm}$ and were used as supports for an immediate fixed provisional restoration replacing the teeth from 15 to 25 . Four more implants were installed in the posterior maxillary regions, but did not present enough initial stability for immediate load. After 30 days, a new surgical procedure was accomplished for the installation of four osseointegrated implants $\left(\right.$ Neodent $\left.^{\circledR}\right)$ in the mandibular anterior region. Over these implants a fixed complete provisional restoration was installed, replacing the teeth from 36 to 46 . The patient is now in the healing phase to allow the beginning of the fabrication phase of the definite restorations.

Effects of adhesive permeability, substrate hydration and polymerization mode on the bond strength of dual resin cements to dentin

Vidotti, H.A.; Carvalho, R.M.; Pegoraro, T.A.

This study investigated how reduction of water flow across the adhesive may affect coupling of resin cements with different curing modes to dentin. Indirect resin crowns were prepared and cemented to exposed, hydrated, flat dentin surfaces of human third molars using Panavia F (PAN) and Bistite II DC (BIS), with either the chemical (CC) or the light (LC) curing mode, as per manufacturers' instructions (control). One experimental group was prepared in a similar way, except that a layer of a non-acidic adhesive resin (Scotchbond Multi-Purpose Plus \#3 - ExSBMP) was placed on the bonded dentin surface before luting procedures. Another experimental group was prepared with additional teeth that were dehydrated in ethanol (ExD) and bonded according to the manufacturer's instructions. Bonded teeth were stored in water for $24 \mathrm{~h}$ at $37^{\circ} \mathrm{C}$, sliced into beams of $0.8 \mathrm{~mm}^{2}$ and tested in tension at $0.5 \mathrm{~mm} / \mathrm{min}$. Means were obtained in $\mathrm{MPa} \pm \mathrm{SD}$ and data analyzed by two-way ANOVA $(\mathrm{p}<0.05)$. Capital letters/luting conditions; lower cases/materials conditions. Mean mTBS values for groups were as follows: CONTROL: PANCC, $25.3 \pm 8.5 \mathrm{Aa}$; PANLC, $23.3 \pm 11.3 \mathrm{Aa}$; BISCC, $26.2 \pm 13.5 \mathrm{Aa}$; BISLC, $33.8 \pm 16.0 \mathrm{Ba}$; ExSBMP: PANCC, $35.6 \pm 15.6 \mathrm{Bb}$; PANLC, $18.7 \pm 9.5 \mathrm{Aa}$; BISCC, $18.4 \pm$ 9.7Aa; BISLC, $31.5 \pm 12.0 \mathrm{Ba}$; ExD: PANCC, $43.1 \pm 11.0 \mathrm{Bc}$; PANLC, $40.6 \pm 12.2 \mathrm{Bb}$; BISCC, $38.9 \pm 12.2 \mathrm{Bb}$; BISLC, $49.9 \pm 11.8 \mathrm{Bb}$. Reduction of water flow across adhesive by application of a non-acidic adhesive may not result in improved bond strength for all dual resin cements. Eliminating water from the substrate seems to be the most effective way to improve coupling of resin cements to dentin, regardless of the curing protocol

Oral rehabilitation by osseointegrated implants in a patient with dental agenesis: case report

Trevisan, L.C.; Brandão, M.R.S.; Ishi, M.M.; Accetturi, F.; Meira, S.H.M.P.A.; Eleutério, R.G.; Bastos, E.L.S.

Dental agenesis is relatively common on daily clinic and its resolution is problematic as far as esthetics s concerned. The osseointegrated implants allow not only a more biological resolution among recent proposals, but also a more esthetic result, mainly when associated with full porcelain crown in this clinical case, a 19 year old patient, searched for dental care looking forward improving her physical esthetic and mastication function. After a rigorous clinic and radiographic examination, it was observed that the maxillary and mandibular first premolars were missing and instead the patient had the primary first molars with complete root resorption. The treatment possibilities were analyzed and, considering the esthetics and the effectiveness of 
the results, the proposal was the installation of osseointegrated implants and subsequently implant-supported full-porcelain crowns. In this case of dental agenesis, the oral rehabilitation with osseointegrated implants and all-ceramic crown appeared as the best treatment modality because it met the patient's esthetic expectations, providing harmonic esthetics, preserving the integrity of the adjacen teeth and avoiding local bone resorption (because of the presence of the implant) and consequent activation and bone remodeling.

\section{The role of myofascial pain in the etiology of chronic headaches}

Bosso, R.; Sanitá, P.V.; Cesnik, R.M.; Júnior, F.G.P.A.

Myofascial pain (MFP) is a regional neuromuscular disorder characterized by the presence of hypersensitive points in the muscles (trigger points - $T r P$ ), being one of the most common causes of chronic orofacial pain. TrP can refer pain to a zone of reference and the patient may feel this pain as a headache. The aim of this study was to evaluate the role of MFP as a contributing factor to the etiology of chronic headaches in 316 patients. Headache diagnosis was established using the guidelines of the International Headache Society. MFP diagnosis was established performing palpation of the masticatory and cervical muscles in order to find $\operatorname{TrP}$ that would reproduce the headache. The results were submitted to percent analysis and showed that $24 \%$ of the patients could be diagnosed with tension type headache (TTH), 14\% migraine, 58,5\% TTH with some episodes of migraine and 3.5\% with other headache types. Furthermore, $74.5 \%$ of the patients could be diagnosed with MFP and in $94.5 \%$ of these patients, headache could be reproduced during palpation of the TrP. Regarding the $\operatorname{TrP}$ that reproduced the headache, $60 \%$ were found in temporalis muscle. TTH was the type of headache with the higher reproducibility. However, migraine could also be reproduced in $58 \%$ of the patients diagnosed with it. The authors concluded that MFP is one of the contributing factors associated to chronic headaches, thus a clinical examination performed to diagnose this disorder would be recommended in chronic headache patients.

Implant-supported metal-ceramic crown on maxillary anterior region: case report

\section{Miyashiro, M.; Suedam, V.}

With the introduction of the "metal free" prostheses, metal-ceramic crowns have been related to several esthetic failures in the maxillary anterior region. In fact, these failures are due to an inadequate planning in metal-ceramic prostheses. The lack of thickness to apply an adequate porcelain layer, associated with lack of dentist's skills, lead to a poor esthetic result. In implant-supported prostheses, the application of an ideal porcelain layer on the cast metal provides a more favorable esthetic effect, making the crown practically imperceptible. The goal of this work was to describe a case in which an implant-supported metal-ceramic crown was made on the maxillary incisor region. Patient missing the maxillary left central incisor due to coronal fracture and posterior root fracture sought treatment. After tooth removal and alveolar bone new formation, the implant was fixed (3i Implant Innovations Inc.). After 6 months of osseointegration, a reopening procedure was performed and, 15 days later, a provisional restoration was made for gingival conditioning providing an adequate gingival contour. After that, color selection and transfer impression were done for the confection of a cemented metal-ceramic crown on a milled prefabricated titanium abutment. Porcelain esthetics was adjusted for shape and texture. After glaze, the crown was fixed with provisional cement. The cemented implant-supported metalceramic crown for maxillary anterior region was well indicated with no esthetic loss for the patient, following the guidelines of esthetics and biology.

\section{Palpation of the lateral pterygoid area on detection of myofascial pain: sensitivity} and specificity

\section{Conti, T.R.; Silva, R.S.; Nunes, L.M.O.; Gelmini, M.; Conti, P.C.R.}

RDC/TMD has been considered the gold standard in research as a way to diagnose TMD and its subgroups. Surprisingly, the intraoral palpation of the lateral pterygoid area is included as one of the muscle palpation sites, despite all the past findings showing that the procedure is anatomically imprecise. The goal of this study was to evaluate the validity of intraoral palpation of the lateral pterygoid area as part of the physical examination protocol to detect myofascial pain disorders, according to the RDC/TMD. Seventy-seven consecutive women, matched for age, were divided into 2 groups: 44 in the myofascial pain group and 33 without TMD signs and symptoms TMD (control group). One calibrated examiner blinded to the groups performed 2 intraoral palpation of the lateral pterygoid area on each side, with a 5-minute interval between palpations. The responses were scored as 1 if negative and 0 if positive. If there was a tie, a third assessment was then performed. The sensitivity, specificity, negative and positive predictive values were calculated for analysis. The test detected $45.45 \%$ of true positives, $9.74 \%$ of false positives, $33.12 \%$ of true negatives and $11.69 \%$ of false negatives. Sensitivity and specificity were calculated as $79.55 \%$ and
$77.27 \%$, respectively. The specificity was bellow the $90 \%$ recommended by the literature, indicating a high number of false positive results. Positive and negative predictive values were $82.35 \%$ and $73.91 \%$, respectively. The method used by the RDC/TMD criteria to make a myofascial pain diagnosis should be revised, based on the findings of the present study, specially the very low specificity. Further investigation is necessary to confirm these results. The authors wish to thank FAPESP - Brazil - Grant \#04/13160-7 for the financial support.

\section{Effect of experimental chewing on the masticatory muscle pain onset}

Leme, V.S.P.; Silva, R.S.; Nunes, L.M.O.; Yassuda, S.; Conti, P.C.R.

Muscle pain is the most prevalent of TMD subtypes. There is no consensus whether or not mastication can produce or aggravate muscle pain. The goal of this study was to analyze the effect of an experimental chewing exercise on the masticatory muscle pain. Twenty-nine women with myofascial pain (RDC/TMD) with an initial visual analogue scale (VAS) above $30 \mathrm{~mm}$, and 15 women, free of TMD signs and symptoms, were selected for this study. Baseline pressure pain thresholds (PPT) was obtained bilaterally on the masseter, and anterior, medium and posterior temporalis muscles. VAS assessed the baseline report of pain intensity. The individuals were then asked to chew a gum (Trident) for nine minutes on the preference side (right or left). Every 3 minutes during chewing a VAS should be scored (VAS3, VAS6 and VAS9). After the exercise (9 minutes), the PPT was recorded one more time. Afterwards, the individuals were submitted to a rest period of another 9 minutes. Additional VAS was scored at 12, 15 and 18 minutes. Right after the rest period, a new PPT assessment was performed. Friedman test and Student-Newman Keuls $(\boldsymbol{\alpha}=0.05)$ were used for the statistical analysis. A significant increase on the pain levels for both VAS and PPT was noted in the experimental group, in comparison to the control group. Pain levels on the TMD patients were higher after the 9 minutes of chewing compared to baseline. The reduction on the VAS scores was more pronounced than the one on the PPT values in the experimental group after the rest period. The present study corroborates the harmful effect of masticatory function on the muscle pain. Therefore, patients should be asked to avoid hard foods or prolonged chewing. The authors wish to thank CNPq-Brazil for the financial support.

Functional and esthetic immediate rehabilitation in an aged patient with osteoporosis

Alves, S.V.; Montandon, A.A.B.; Fais, L.M.G.; Tibério, D.; Santos, M.T.B.R.; Pinelli, L.A.P.

The treatment of an aged patient with dental integrated requirements demands a multidisciplinary and individual vision that is planning for your reality. The patient O.V.C., 70 years old, with osteoporosis diagnosis, looked for dental treatment because of dissatisfaction with her chewing efficiency and esthetics, muscular fatigue, temporomandibular joint pain, in addition to pain in spinal column and legs. The aim of this study was to show the specificity and importance of an exam that includes an evaluation of the systemic condition, use of medicaments, cognition (Mini Mental State Examination), depression level (Geriatric Depression Scale) and dental evaluation in the proposed and executed treatment. After rigorous clinical interview, it was observed that the patient did not present indications of cognitive compromising or depression, but she was very anxious for an improvement of the functional and esthetic conditions. There was the need of recovery of the reverse curve of the smile, vertical dimension of occlusion and accentuate wear in the teeth of her removable dentures. However, due to the strong pain, the treatment needed to be accomplished in four sessions. The treatment involved periodontal procedures, recovery of the vertical dimension of occlusion with acrylic resin on the posterior teeth of her removable dentures, cosmetic dentistry for incisal guide determination, and placement of acrylic resin veneers in the anterior teeth of the maxillary denture. It was concluded that, with the proposed treatment, the smile and the patient's chewing efficiency was recovered temporarily in a satisfactory way, contributing to a better planning of the definitive rehabilitation and with positive results for the patient's quality of life.

Stress distribution in implant-supported RPD: periodontal ligament influence on internal stress distribution - FEA analysis

Archangelo, C.M.; Pereira, J.A.; Junior, M.M.; Junior, A.C.F.; Rocha, E.P.

The application of diverse hypotheses in a finite element analysis (FEA) model has been critical to obtain precise results in the treatment with implant-supported removable partial denture (RPD). In view of this, the aim of this study was to assess, by means of a two-dimensional FEA, the internal stress distribution of a distal extension RPD (DERPD) associated with an osseointegrated implant, considering the homogenous and non-homogenous reproduction of the periodontal ligament (PL). For such purpose, 6 models (M) were created, representing the following: MA - hemi-arch containing tooth 33 only and the edentulous distal alveolar ridge; MB - similar to MA, but with DERPD replacing the absent teeth; MC - similar to MB, 
with an implant (3.75 x $10.0 \mathrm{~mm}$ ) supporting the denture in the posterior region; MD, ME and MF - similar to models A, B, and C respectively, except for PL, which was modeled in a non-homogeneous form, considering the following 4 groups of fibers: crest, horizontal, oblique and apical. Like this, as result, the maximum stres concentrations (in MPa) were as follows: MA (58.976); MB (77.863); MC (207.195); MD (110.33); ME (191.36) and MF (326.60); the non-homogeneous PL highlighted the benefits of the implant, drastically reducing the stresses on the posterior $1 \frac{1}{2}$ of the alveolar ridge; furthermore, the variation in the acting stress values was lower under the non-homogeneous condition. The conclusions were: The PL in the nonhomogeneous form emphasized the benefits of the osseointegrated implant in relation to the abutment structures in a more pronounced manner than the homogeneous condition, and is essential for obtaining results that corroborate the previous clinical data as the maximum stress values, the variations among the models and the internal stress distribution were found to be in agreement with those established by the literature.

Pain and electrical activity of anterior temporal and masseter muscles across menstrual cycle in oral contraceptive users and non users

Batista, F.R.S.; Garcia, A.R.; Sundefeld, M.L.M.M.; Zuim, P.R.J.; Mori, G.G.; Turcio, K.H.L.

Electric activity and pressure-pain threshold of the temporal and masseter have been used in the diagnosis of some disorders that cause pain, such as the temporomandibular disorders (TMD). As the majority sufferers of the TMD are women, it is possible that hormone fluctuation across menstrual cycle predisposes to this disorders. The aim of this study was to analyse the electrical activity and pressurepain threshold of anterior temporal and masseter across menstrual cycle. Twentyeight women at reproductive age were selected to the research. Thirteen were ora contraceptive users, and 15 were non users. All of them answered a questionnaire and were submitted to electromyographic exams and algometry of the muscles during three consecutive menstrual cycles. The results permitted to verify that pressurepain threshold and the electrical activity of both muscles during rest and during chewing did not demonstrate statistically significant difference across menstrual cycle. However, work side temporal activity was significantly more elevated in ora contraceptive users than in non users. It may be concluded that pressure pain threshold and electrical activity did not vary during different phases of menstrual cycle, bu anterior temporal muscle demonstrated significantly higher activity in oral contraceptive users.

\section{Parafunctional habits and the stomatognathic system}

Cunha, C.O.; Lima, R.T.; Bonfante, G.

The functional activities of the stomatognathic system are described as chewing, swallowing, phonation and breathing. However, part of these activities is parafunctional. This kind of parafunctional activity damages the structures of the system from the moment at which it exceeds the individual's physiologic tolerance. Likewise, physiologic tolerance and signals and symptoms that parafunctional activity can cause vary for each person. It is also known that these habits can be destructive in terms of physical, emotional and/or social effects. The parafunctional habits $(\mathrm{PH})$ can occur during awakeness and/or during sleep. During awakeness, the habits are accomplished, generally, unconsciously and include tooth clenching and grinding, lip, tongue and cheek biting, chewing gums and drops, nails and objects biting, tongue thrusting and jaw play. During sleep, the unique PH accomplished is bruxism. During the last decades, several authors have demonstrated that damages to the stomatognathic system affect mostly teeth, periodontium, muscles and temporomandibular joint (TMJ). Recently, in 2005, Glaros et al. ratified that dental wearing and/or fracture occurs due to prolonged occlusal contact accomplished during tooth clenching and grinding, or also due to biting of nails and objects. These PH can damage periodontal tissues, which can react with dental movements and progressive mobility. In 2006, Winocur stated that PH are risk factors for temporomandibular disorders. Damages to masticatory muscles and TMJ, separately or conjunctly, were related to $\mathrm{PH}$ of chewing gum and jaw play, among others. Studies noted that there is a great relation among habits and the emotional tensions, mainly stress and anxiety. The aims of this work were to present the identification of $\mathrm{PH}$ and the damages that PH can cause to the stomatognathic system, and suggest means to diminish or eliminate the occurrence of $\mathrm{PH}$ and their possible damages.

Periodontology and Prosthesis integration on the planning of implant-supported dentures and fixed partial dentures, aiming at excellent anterior esthetics

Ferreira, F.M.; Novais, V.R.; Júnior, P.C.S.; Prado, C.J.; Nóbilo, M.A.A.; Neto, A.J.F.

Patients are more demanding with dental treatment in relation to esthetic, functional and biological aspects. Therefore, a comprehensive analysis about teeth, lips, gingiva and face is necessary to oral rehabilitation success and to provide a harmonic smile.
The interaction of gingival tissues and teeth is fundamental to optimal esthetics of prostheses when the anterior superior region is involved. Thus, care with periodontal tissues is important in prosthetic planning to obtain esthetics, function, better hygienic and good phonetics. The formation of interdental or interimplant papilla and the reconstitution of gingival concave arch are goals of current Dentistry in order to optimize the final esthetic aspects in implant-supported dentures and fixed partial dentures, making that the prosthetic pieces mimic the natural dentition in relation to their emergence profile. The aim of this work was to discuss several techniques of planning, such as gradual pressure, surgical techniques and artificial gingiva, aimed at achieving a better relationship between periodontal tissues and prostheses.

\section{Occlusal adjustment after orthodontics: case report}

Camargos, G.V.; Neto, A.J.F.; Rodrigues, M.M.; Silva, M.R.

Oclusal adjustment has had a long and troubled history because of empirical approaches owing to unfamiliarity with the acceptable biological principles (total function of the stomatognathic system), introduction of the concept of bilateral balanced occlusion and practice of prophylactic adjustment. The therapeutic behavior for oclusal adjustment considers modifications in the occlusion (on the surfaces of teeth, restorations or prostheses) by means of selective dental tissue removal or addition of restorative materials, seeking to harmonize the functional aspects of the maxillomandibular complex in centric relation occlusion and the eccentric movements. This technique is indicated when signs and symptoms of traumatic occlusion are present and the occlusal relations can be improved by the adjustment, before extensive restorative treatments, and for stabilization of the outcomes of orthodontic treatment and oral and maxillofacial surgery. In this context, the patient of this case presented signs of bone and dental structure loss (abfraction and wear facets) resulting from a traumatic occlusion after orthodontics. Thus, with the primary objective of improving the functional relations of the dentition, so that the patient could undergo periodontal esthetic surgery, oclusal adjustment was indicated to establish the anterior guide in the mandibular eccentric movements, eliminating the occlusal interferences. This approach was preceded by the clinical stages of taking impressions, obtaining the study models for further assembly in a semi-adjustable articulator, and mapping the interferences in the articulated models. After completion of the adjustment, canine guides were obtained as well as oclusal stability.

Interrelation between Prosthodontics, orthognathic surgery, and dental implants in oral rehabilitation of subjects with cleft palate

Andrade, P.C.A.R.; Pinto, J.H.N.; Mazzotini, R.; Lopes, J.F.S.

Reverse planning has an extreme importance on implant-supported prosthesis. Planning gives subsidies necessaries to evaluate ways to manage and execute treatments. A case report evaluating the importance of reverse planning in complex cases, involving Prosthodontics, dental implants and orthognathic surgery is presented. A 39-year-old female patient with cleft palate and severe atrophic upper jaw, edentulous upper and lower jaws was seen at the dental service of the HRACUSP four years ago needing implants and fixed prosthesis to prepare for a future orthognathic surgery. All remaining teeth were removed and 6 upper and 4 lower implants were installed with the purpose of installing upper and lower fixed prosthesis. After implant transference and during trial denture the amount of resin and the esthetics and phonetics of the patient were evaluated. After bite recording, cast models were mounted in semi-adjustable articulator (SAA) and prostheses were made having the discrepancy corrected. Prostheses were inserted and impressions obtained. Cast models were mounted again in SAA in order to accomplish surgical planning. During orthognathic surgery, upper and lower jaws were manipulated until assuming an ideal position similar to the planned one. Two surgical guides were confectioned (upper and lower jaws were manipulated until assuming an ideal position similar to the planned one). Three months after surgery, a new upper interim prosthesis was fabricated in order to improve esthetics and occlusion. In this case, $90 \%$ surgical, the reverse planning was extremely important to define the treatment plan and not to raise false expectations to the patient with only the prostheses and dental implants.

\section{Tooth-implant union in oral Prosthodontics: report of two cases}

Paula, G.A.; Neves, F.D.; Prado, C.J.; Mota, A.S.

Prostheses with tooth-implant union are a reason of controversy in Dentistry and require more studies. However, in certain clinical situations, these prostheses are employed in the same cost-effectiveness level as that of other prosthetic solutions. Thus, the patient, duly informed of the complexity of its clinical situation, will opt for the best treatment modality, and the tooth-implant union may be the most indicated. In this context, this work reports two longitudinal follow ups (8 and 5 years) of prosthetic rehabilitation, showing the success of this type of union. In the first case, 
3 implants were placed in the region of the spaces of teeth 21 and 22 and reestablished the Roy Polygon in association with natural teeth of other plans. In the second case, 3 implants were installed and added to the natural teeth, in a type of implant-supported denture based on force distribution on the Roy Polygon. It is important to point out that, in the first case, the treatment options were: isolation of posterior teeth (tota crown), bone graft, and cantilevers of two previous teeth (11 and 12) or 6-element partial removable denture (PRD). In the second case, a class I PRD could have been fabricated, with tooth 14 being a direct pillar with 1:1 crown/root ratio, and separation of the segments. The construction of implant-supported or tooth-supported dentures alone was not compatible with the patient's economical conditions. Therefore, it is important that dental examination includes evaluation of the type of pillar, soft tissue and bone support to be used. Situations similar to those reported in these cases may appear in the clinical practice and will raise the question: prosthesis with toothimplantation union "cannot or should not" be used as solutions in certain cases oral rehabilitation?

\section{Reconstruction of the smile with laminated porcelain veneers}

Camargo, A.B.; Camargo, J.A.R.; Archangelo, C.M.

The greatest challenge when establishing esthetic excellence is to create harmony of the teeth with the oral components without leaving aside the knowledge and application of specific principles. This avoids the fabrication of prosthetic piece out of the esthetic standards and clinical failure. The present study presents a case of a 53-year-old male patient who needed esthetic modification of the maxillary anterior region. The maxillary anterior teeth were in good clinical conditions but had compromised anatomy. Teeth 13,11, 21 and 23 had large diastemas. Teeth 12 and 22 presented worn edge. In addition, the mesial of the teeth 11 and 21 had old fillings previously done in an attempt to close the spaces. The line formed by the incisal border was not compatible with the lower lip and teeth. In contrast with the negative space, they were not in harmony, compromising the oral esthetics and causing a negative visual impact. For planning and diagnosis, study models were obtained. The patient was submitted to a simulation of the final result (Test Drive) by a direc reproduction of the previously obtained study models. The proposed treatment was in-office bleaching associated with at-home bleaching and fabrication of laminated porcelain veneers (IPS Empress II) for teeth 13, 12, 11, 21, 22 and 23. The final veneers reproduced the color, texture and optical qualities of the dental enamel in total harmony with the antagonistic arch, oral region and the patient's face as a whole. Therefore, it may be concluded that it is possible to reach esthetic and functional success in the anterior dental region as far as basic principles are respected during treatment planning.

\section{Public Health}

\section{Evaluation of oral health importance according to the perception of hospitalized} patients

\section{Fernandes, L.A.; Lima, D.C.; Saliba, N.A.; Garbin, C.A.S.}

The presence of dentists in hospital settings is the result of the progresses reached in the formation of a multidisciplinary and integrated team with the goal of offering to the hospital users a larger number of health services. The aim of this study was to evaluate the importance of oral health according to the perceptions of hospitalized patients in a specialized trauma service in the city of Araçatuba, Brazil. 64 patients were enrolled in this descriptive and transversal study. An open-ended questionnaire was used for data collection and later the analyses were conducted using Epi-Info 2000 v.6.04 statistical program. The results showed that there was a predominance for the 21-30-year-old age range (40.62\%) and male population (68.75\%). Half of patients affirmed that they had their last dental visit within the past 6 months to 1 year due to periodontal problems (35\%) and caries (20\%). Regarding the self importance of teeth, $50 \%$ reported esthetics, $34.38 \%$ mentioned chewing and feeding, $6.25 \%$ cited function and $9.37 \%$ answered that the teeth are "the life equilibrium". Most respondents (90.63\%) believed that the role of the dentist in a hospital setting would be "the care of teeth". It may be concluded that all patients considered oral health important and believed that the presence of a dentist in the hospital staff would be important to help in the promotion of integral health.

\section{Educative tasks for schoolchildren in the state of Rondônia: experience report}

Xavier, A.; Pimentel, T.F.; Simões, R.C.S.; Silva, R.P.R.; Merlini, S.P.; Bastos, J.R.M.

The present study aims at presenting an educative-preventive experience work carried out at Mato Grosso School in the city of Monte Negro/RO with primary schoolchildren aged 6 to 10 years. The tasks were carried out in a playful manner in order to allow the children having a better understanding of the tasks. Firstly, an educative theater play was performed focusing on oral health and speech, language and hearing health, with special emphasis on brushing techniques, non-carious diet, general hygiene techniques and guidelines on hearing, vocal health and deleterious habits. Secondly, a recreational educative activity was performed in which a track was used to ask questions about oral and speech, language and hearing health, based on the themes that had been brought up during the theater play. The winner would be the team that walked more spaces of the track, which was indicated by means of a dice, as the participants answered to the questions correctly. After these educative activities, all children performed supervised toothbrushing, separating them by room and using the Bass technique to guide the process. A total of 337 children took part in this activity, receiving, in a playful manner, instructions on oral and speech, language and hearing health during the class period, thus adding extra information to the activities that are part of the beginning of the school year. It may be concluded that the activities provided a greater level of instruction on oral and speech, language and hearing health to the children, who absorbed the information with a high degree of accuracy of the questions and also appeared s quite motivated to do the toothbrushing.

Influence of primary attention and infantile growth on dental caries in preschool children from Borebi, SP

Silvério, M.A.; Oliveira, M.F.S.; Lauris, J.R.P.; Tomita, N.E.

Dental caries is strongly associated with the socioeconomic conditions. In addition to receiving little oral health care, children from low-income families can also have growth deficits. The objective of this study was to evaluate the influence of the socioeconomic level on oral health conditions and infantile growth in preschool children. The targets of this article were children aged 4-6 years living in Borebi, SP, Brazil. During the visits to these children, the following activities were performed: oral examination, using oral mirror and periodontal probe (WHO 1997); socioeconomic evaluation of the families using a questionnaire answered by parents/ guardians; anthropometric measures were taken (height was measured with a tape measure in millimeters and weight was determined with a digital weighing-machine accurate to 100 g). 75 children were examined of which $46.67 \%$ were female and $53.33 \%$ were male; $92 \%$ attended preventive programs; $50.67 \%$ were caries free and the DMF-T index was 1.83 . No statistically significant association was observed between the socioeconomic level, infantile growth and dental caries. There was statistically significant negative correlation $(\mathrm{p}<0.05)$ between height/age index and DMF-T index, denoting that the more favorable the child growth, the better the oral health conditions. However, it is important to highlight that $92 \%$ of the children attend preventive programs in their schools and that the city has $100 \%$ of coverage of the Government's Family Health Program, with important repercussions on infantile health situation.

Epidemiology of dental caries in adolescence: influence of primary attention

Oliveira, M.F.S.; Silvério, M.A.; Lauris, J.R.P.; Tomita, N.E.

Many variables have been associated with the occurrence and distribution of dental caries. The objective of this study was to evaluate the influence of the socioeconomic variable on oral conditions and growth in adolescents aged 11, 12 and 13 years in the city of Borebi, SP. The development of oral health actions in the primary attention level and their repercussion on dental caries prevalence was also verified. The adolescent growth (height and weight measures) and its association with dental caries were also evaluated. Home visits were done and, after granting parental consent, a socioeconomic questionnaire was applied as well as oral examination and anthropometric measures of the adolescents were done. 108 adolescents were examined and a mean DMF-T $=2.29$ was obtained. Statistically significant associations between family income and adolescent anthropometric measures were found $(p<0.05) .21$ examined adolescents were caries-free and 24 of them presented DMF-T $=1$, this was the most frequent index recorded. $40 \%$ of the visited families had a mean monthly income between 1 or 2 minimum salaries. These findings demonstrate the effectiveness of the primary attention to oral health, as most of adolescents presented low DMF-T, even those belonging to families with low socioeconomic conditions. Maximum DMF-T found was 8, in only 3 adolescents; however they had recently moved to the city and were not attending any preventive programs before. It is important to highlight that $98.15 \%$ of the examined adolescents attended preventive programs in their schools and that the city has $100 \%$ of coverage of the Government's Family Health Program, with important repercussions on the adolescents' view of health. 